\title{
Eine genaue und vielseitig anwendbare Vorrichtung zur Erzeugung von Flüssigkeitsgradienten
}

\author{
HARRY SVENSSON UAd ALFRED FORCHHEIMER \\ Abteilung für physikalische Chemie, Jubileumslaboratorium der Wallenberg-Stiftung, \\ Karolinska Institutet, Stockholm, Schweden
}

Herrn Professor Dr. Ole Lamm zum 60. Geburtstag gewidmet

\begin{abstract}
Es wird eine Mischvorrichtung zur Erzeugung von Gradienten in Flüssigkeiten beschrieben. Sie besteht aus mehreren geschlossenen, luftfreien, in Serie geschalteten, kapillar verbundenen und mit Rührkörpern versehenen Kammern, alle mit demselben freien Volumen. Das ganze Kammersystem ist in durchbohrten Kugelschliffen gelagert und wird mittels eines Motors in Rotation gesetzt, während die Rührkörper auf den zylindrischen Kammerwänden frei rollen. Durch Wahl verschieden grosser Rührkörper kann das Gesamtvolumen des Kammersystemes leicht verändert werden. Die Volumengleichheit der Kammern macht die mathematische Berechnung der Ausflusskonzentration besonders einfach und die allgemeine Lösung der Differentialgleichung eines Systemes mit beliebiger Anzahl von Kammern wird gegeben. Obgleich die Lösung eine Exponentialfunktion ist, wird gezeigt und durch Versuche bestätigt, dass sehr genau lineare Ausflussfunktionen (konstante Gradienten) erhalten werden können, falls die Ausgangskonzentrationen passend gewählt werden. Die luftfreie Füllung des Kammersystemes gewährt eine ausserordentlich gute Reproduzierbarkeit, und eine vollkommene Übereinstimmung der Ausflussfunktionen mit der Theorie wird ebenfalls bestätigt.
\end{abstract}

Dhysikalisch-chemische Prozesse mit Anwendung veränderlicher Flüssigkeitseigenschaften, z. B. mit Hinsicht auf Polarität, Dichte, $\mathrm{pH}$, Leitfähigkeit u. s. w., gewinnen mehr und mehr an Bedeutung. Als Folge davon steigen die Anforderungen an Mischvorrichtungen ständig. Viele solche Vorrichtungen sind bereits beschrieben worden. Die meisten davon haben jedoch eine schlechte Reproduzierbarkeit oder ein allzu begrenztes Anwendungsgebiet. In der Absicht, eine Mischvorrichtung zu konstruieren, welche so vielen experimentell bedingten Anforderungen wie möglich genügt, haben die Verfasser die wünschenswerten Eigenschaften einer solchen Vorrichtung in folgenden acht Punkten zusammengefasst. 
1) Die Vorrichtung soll eine vielseitige Gestaltung der Funktion $P(v)$ zulassen, wobei $v$ das von der Vorrichtung vom Startaugenblick an abgegebene Flüssigkeitsvolumen und $P$ die stetig veränderliche Eigenschaft darstellen. Die Möglichkeit, eine lineare Funktion $P(v)$, d. h. einen konstanten Gradienten $P^{\prime}(v)$ zu erzeugen, wird als besonders wichtig betrachtet.

2) Die Vorrichtung soll eine bequeme Variation des Gesamtvolumens zulassen, innerhalb dessen sich die Eigenschaft $P$ verändert.

3) Die Funktion $P(v)$, welche von der Vorrichtung geliefert wird, soll von allen anderen Eigenschaften der Flüssigkeitskomponenten unabhängig sein. So soll beispielweise ein Polaritätsgradient von der Dichte der Flüssigkeitskomponenten, und ein Dichtegradient von einer eventuellen Verschiedenheit der Viskosität unabhängig sein.

4) Es soll möglich sein, den Verlauf der Funktion $P(v)$ mit Hilfe der Ausgangsbedingungen zu berechnen und umgekehrt, geeignete Ausgangsverhältnisse aus einer bestimmten gewünschten Form der Funktion $P(v)$ zu ermitteln.

5) Die Vorrichtung soll genau und reproduzierbar arbeiten, $d . h$. bei wiederholten Versuchen unter denselben Ausgangsbedingungen genau dieselbe Funktion $P(v)$ liefern. Diese Funktion soll ausserdem auch von der Ausflussgeschwindigkeit aus der Vorrichtung unabhängig sein.

6) Die Vorrichtung soll für chromatographische Elution hinreichend langsam und zur Bereitung von stabilisierenden Dichtegradienten genügend schnell arbeiten können.

7) Die', Bedienung der Vorrichtung soll bequem und die Instandhaltung leicht sein.

8) Die Vorrichtung soll, sei es dass sie kommerziell oder in der Laborwerkstatt hergestellt werde, zu einem Preis erhältlich sein, welcher zu dem Anwendungsgebiet und einem gewöhnlichen Laboratorienbudget in angemessenem Verhältnis steht.

Ein Studium der meist angewendeten Mischvorrichtungen zeigt deutlich, wie schwer es ist, alle diese Anforderungen gleichzeitig zu erfüllen. So ergibt beispielweise die Zusammenschaltung einer geschlossenen Mischkammer mit einem offenen Behälter nur eine einzige (exponentielle) Form der Funktion $P(v)$, und das anwendbare Flüssigkeitsvolumen ist durch den Rauminhalt der Mischkammer bestimmt. Andererseits genügt eine solche Vorrichtung den Anforderungen in den Punkten 3) bis 8). Eine Vorrichtung mit zwei parallelgeschalteten Kammern, die eine mit nach oben zunehmendem und die andere mit abnehmendem Querschnitt, ist für spezielle Zwecke anwendbar, genügt aber nicht den Anforderungen in den Punkten 1), 3) und 5). Eine Anordnung mit zwei in Serie geschalteten offenen Zylindern, von denen der eine mit Rührwerk versehen ist, wird häufig angewendet und genügt den Anforderungen 1), 2), 4), 6), 7) und 8). Der resultierende Gradient ist jedoch von Unterschieden mit Hinsicht auf Dichte und Viskosität abhängig und ändert sich ausserdem mit der Ausflussgeschwindigkeit. Peterson und Sober's ${ }^{1}$ Apparat "Varigrad" * arbeitet nach diesem Prinzip, doch mit neun in Serie geschalteten, mit Rührwerk versehenen zylindrischen Gefässen. Die grosse Anzahl der Gefässe lässt eine praktisch genommen unbegrenzte Formbarkeit

* Hersteller: Buchler Instruments Inc., 514 West 147th Street, New York, N. Y. 
der Funktion $P(v)$ zu; die Abhängigkeit von Dichte, Viskosität und Ausflussgeschwindigkeit bleibt jedoch bestehen. Bisher beschriebene Methoden zur Bereitung von Flüssigkeitsgradienten wurden von Peterson und Sober ${ }^{1}$ sowie von Svensson ${ }^{2}$ zusammengestellt.

Sämtliche Mischvorrichtungen mit offenen Behältern weisen Abhängigkeit von Dichte- und Viskositätsunterschieden auf. Um die wichtige Anforderung 5) zu erfüllen, haben die Verfasser deshalb eine Ausführung mit geschlossenen und mit Rührkörpern versehenen Kammern gewählt, wobei die Luft völlig aus dem Kammersystem ausgeschlossen ist. Die Wahl eines Systemes mit mehreren Kammern ist notwendig, um der Anforderung 1) zu genügen, weil eine einzige Kammer nur eine unveränderliche Exponentialfunktion liefert. Weiterhin wurde für sämtliche Kammern das gleiche freie Flüssigkeitsvolumen gewählt, da dies die mathematischen Berechnungen in Zusammenhang mit den Anforderungen in Punkt 4) bedeutend erleichtert. Mit Hinsicht auf Punkt 2) wurde eine Konstruktion gewählt, in welcher austauschbare Rührkörper es möglich machen, das freie Flüssigkeitsvolumen $\mathrm{zu}$ verändern. Wie die übrigen hier aufgestellten Anforderungen erfüllt werden geht aus der Beschreibung der Apparatur hervor.

THEORETISCHE GRUNDLAGEN VON MISCHVORRICHTUNGEN, BESTEHEND AUS EINER BELIEBIGEN ANZAHL IN SERIE GESCHALTETER, MIT RÜHRKÖRPERN VERSEHENER MISCHKAMMERN GLEICHEN RAUMINTHALTES

Der Verlauf der Konzentration des Ausflusses aus einer Mischvorrichtung mit einer einzigen, geschlossenen und mit Rührkörper versehenen Kammer wird durch die wohlbekannte Gleichung:

$$
c_{1}(x)=c_{0}+\left(c_{1}-c_{0}\right) \mathrm{e}^{-x}
$$

dargestellt, wobei $x$ das Verhältnis zwischen dem abgegebenen Flüssigkeitsvolumen $v$ und dem freien Kammervolumen $V$ darstellt, das heisst:

$$
x=v / V
$$

$c_{0}$ ist die der Mischvorrichtung zugeführte konstante Konzentration der einen Komponente, und $c_{1}$ ist die Ausgangskonzentration derselben Komponente in der Kammer.

Zwei geschlossene Kammern des gleichen freien Rauminhaltes $V$, mit Umrührern versehen, in Serie geschaltet und kapillar verbunden, ergeben folgenden Konzentrationsverlauf des Ausflusses:

$$
c_{2}(x)=c_{0}+\left(c_{2}-c_{0}\right) \mathrm{e}^{-x}+\left(c_{1}-c_{0}\right) x \mathrm{e}^{-x}
$$

wobei $c_{2}$ die Ausgangskonzentration in der zweiten Kammer bedeutet. Diese Gleichung wurde zuerst von Drake ${ }^{3}$ aufgestellt und deren Herleitung kann in ihren Einzelheiten aus seiner Schrift oder aus einer späteren Veröffentlichung von Svensson ${ }^{4}$, entnommen werden.

Die gleiche Methodik des Berechnungsganges, auf eine dritte Mischkammer angewendet, liefert die Gleichung:

$$
c_{3}(x)=c_{0}+\left(c_{3}-c_{0}\right) e^{-x}+\left(c_{2}-c_{0}\right) x \mathrm{e}^{-x}+\left(c_{1}-c_{0}\right)\left(x^{2} / 2\right) \mathrm{e}^{-x}
$$

Acta Chem. Scand. 16 (1962) No. 9 
Dies lässt vermuten, dass die Konzentration des Ausflusses aus der $n$ :ten Mischkammer von der Gleichung

$$
c_{n}(x)=c_{0}+\mathrm{e}^{-x} \sum_{i=1}^{i=n}\left(c_{i}-c_{0}\right) \frac{x^{n-i}}{(n-i) !}
$$

wiedergegeben werden kann. Die Richtigkeit ist leicht mit Hilfe des mathematischen Induktionsbeweises nachweisbar. Die Differentialgleichung der Kammer Nummer $(n+1)$ lautet:

$$
c_{n}(x) \mathrm{d} x-c_{n+1}(x) \mathrm{d} x=\mathrm{d}\left(c_{n+1}(x)\right)
$$

und hat die allgemeine Lösung:

$$
c_{n+1}(x)=\mathrm{e}^{-x} \int c_{n}(x) \mathrm{e}^{x} \mathrm{~d} x
$$

welche eine Rekursionsformel darstellt. Setzt man $c_{n}(x)$ aus Gleichung (5) ein, so erhält man dieselbe Gleichung mit $n+1$ statt $n$. Die Gleichung (5) liefert differenziiert folgenden Ausdruck für den Ausflussgradienten:

$$
c_{n}{ }^{\prime}(x)=\mathrm{e}^{-x} \sum_{i=1}^{i=n}\left(c_{i-1}-c_{i}\right) \frac{x^{n-i}}{(n-i) !}
$$

Die Herstellung eines konstanten Gradienten. Gewisse einfache Mischvorrichtungen mit mangelnder Reproduzierbarkeit sind imstande, im Wesentlichen lineare Funktionen $P(v)$ über das ganze zugängliche Konzentrationsintervall zu liefern. Mit geschlossenen, seriengeschalteten Kammern ist eine komplette Linearität nicht zu erhalten, was aus der Gleichung (5) hervorgeht. Unabhängig von der Natur der Polynome von $x$, nähert sich die Funktion asymptotisch dem Wert $c_{0}$, was in diesem Bereich einen konstanten, nicht verschwindenden Gradienten ausschliesst. Dass wir trotzdem geschlossene Kammern gewählt haben, beruht darauf, dass wir gute Reproduzierbarkeit und hohe Genauigkeit höher schätzen als die Fähigkeit, eine vollständige Linearität zu geben. Es wird jedoch im Folgenden gezeigt, dass praktisch genommen konstante Gradienten über mehr als $70 \%$ des zur Verfügung stehenden Konzentrationsunterschiedes erreicht werden können, wenn die Anzahl der Kammern 5 oder mehr ausmacht und die Ausgangskonzentrationen sorgfältig gewählt werden. Nachdem konstante Gradienten für viele Anwendungen ausserordentlich wünschenswert sind, lohnt es sich, die Annäherungsmöglichkeiten für Anordnungen nach den hier beschriebenen Prinzipien näher zu untersuchen.

Man versteht unmittelbar, dass Ausgangskonzentrationen, welche eine aritmetische Reihe bilden, im ersten Teil des Ausflusses einen annähernd konstanten Gradienten geben müssen. In diesem Falle ist der Konzentrationsunterschied zwischen den Kammern:

$$
\Delta c_{i}=c_{i-1}-c_{i}
$$

durch das ganze System konstant, und man erhält für den Gradienten die Gleichung:

$$
c_{n}{ }^{\prime}(x)=\Delta c \mathrm{e}^{-x} \sum_{i=0}^{n-1} \frac{x^{i}}{i !}
$$


die bereits im Jahre 1960 von einem der Verfasser ${ }^{2}$ aufgestellt wurde. Da das Polynom in dieser Gleichung die Potenzreihe von $\mathrm{e}^{x}$, am $n$ :ten Glied unterbrochen, darstellt, wird der Gradient mit wachsender Anzahl Kammern mehr und mehr konstant und nähert sich dem Wert $\Delta c$.

Die Konzentrationen und Konzentrationsgradienten, welche man erhält, wenn man Ausgangskonzentrationen in aritmetischer Reihe anwendet, sind in Abb. 1 bis zu sechs Kammern graphisch dargestellt.

Im Folgenden wird das Ergebnis von Versuchen, mit einer Vorrichtung beschriebener Art einen konstanten Gradienten zu erreichen, quantitativ durch den Teil des zur Verfügung stehenden Konzentrationsunterschiedes ausgedrückt, innerhalb dessen der Gradient weniger als $\pm 10 \%$ von einem Mittelwert abweicht. Im vorliegenden Diagramm wurden diese Bruchteile erhalten, indem man von einer Waagrechten $c^{\prime}(x)=9 / 11$ ausging und von deren Schnittpunkten mit den Gradientkurven lotrechte Linien bis zum Schnitt mit den entsprechenden Integralkurven zog. Die Konzentrationsintervalle zwichen Null und diesen Punkten stellen die Gebiete dar, in welchen die Konzentration, gemäss oben gegebener Definition, hinreichend linear verläuft. Dieses Gebiet wächst mit zunehmender Anzahl von Kammern erst stark und dann immer langsamer an. Für eine Ein-Kammer-Mischvorrichtung werden die Linearitätsabweichungen schon bei $0,18 c_{0}$ grösser als $\pm 10 \%$, während ein SechsKammer-System ein bis auf $\pm 10 \%$ lineares Gebiet von $0,60 c_{0}$ aufweist.

Es ist faktisch möglich, die Linearität der Konzentrationsfunktion noch zu verbessern, wenn man von dem Prinzip der Anwendung von Ausgangskonzentrationen in aritmetischer Reihe absteht. Die Gleichung:

$$
\mathrm{e}^{x} c_{n}^{\prime \prime}(x)=\Delta c_{1} \frac{x^{n-1}}{(n-1) !}+\sum_{i=1}^{n-1}\left(\Delta c_{i+1}-\Delta c_{i}\right) \frac{x^{n-i-1}}{(n-i-1) !}=0
$$

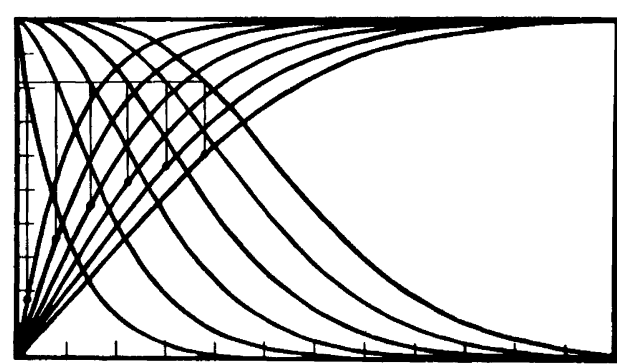

Abb. 1. Ausflusskonzentrationen (steigende Kurven) und deren Gradienten (fallende Kurven) von einem Kammersystem mit einer bis sechs Kammern als Funktion des gelieferten Volumens in Einheiten des Kammervolumens, für den Fall, dass die Ausgangskonzentrationen eine aritmetische Reihe bilden.

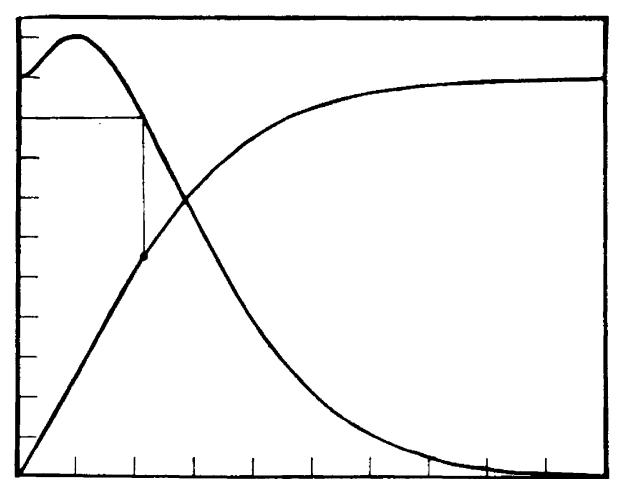

Abb. 2. Ausflusskonzentration (steigende Kurve) und deren Gradient von einem Kammersystem mit drei Kammern als Funktion des gelieferten Volumens, für den Fall, dass die vier Ausgangskonzentrationen den Zahlen 4, 2, 1 und 0 proportional sind. (Vgl. die Gleichungen 12 bis 16.)

Acta Chem. Scand. 16 (1962) No. 9 
gibt die $x$-Werte für welche der Konzentrationsverlauf Inflexionspunkte aufweist. Die Gleichung ist $(n-1)$ :ten Grades mit Koeffizienten, welche von den Unterschieden der Ausgangskonzentrationen gebildet werden. Es ist offenbar möglich, die Anfangskonzentrationen so zu wählen, dass die Gleichung (11) $n-1$ reelle Wurzeln für vorherbestimmte $x$-Werte hat. Wählt man diese Punkte sorgfältig, kann eine Konzentrationskurve erreicht werden, welche mit derartig kleinen Amplituden um eine gerade Linie spielt, dass sie kaum von einer solchen unterschieden werden kann. Dieses Verfahren wird hier durch einige Beispiele erläutert.

Für drei Kammern in Serie nimmt die Gleichung (11) folgende Form an:

$$
\Delta c_{1}\left(x^{2} / 2\right)+\left(\Delta c_{2}-\Delta c_{1}\right) x+\left(\Delta c_{3}-\Delta c_{2}\right)=0
$$

Werden Inflexionspunkte bei $x=0$ und $x=1$ gewählt, muss diese Gleichung mit $x(x-1)=0$ identisch sein. Hieraus erhält man die Beziehungen:

$$
\Delta c_{3}=\Delta c_{2} \text { und } \Delta c_{1}=2 \Delta c_{2}
$$

Für die Ausgangskonzentrationen in beliebigen Einheiten sind dann die Zahlenfolgen

$$
4,2,1,0 \text { und } 0,2,3,4
$$

möglich, wobei sich die eine Zahlenfolge auf einen positiven und die andere auf einen negativen Gradienten beziehen. Werden diese Ziffern in die Gleichungen (5) und (8) eingesetzt, erhält man folgenden Konzentrationsverlauf:

$$
c_{3}(x)=\left(c_{0} / 4\right)\left(x^{2}+3 x+4\right) \mathrm{e}^{-x}
$$

und den Gradienten:

$$
c_{3}{ }^{\prime}(x)=\left(c_{0} / 4\right)\left(x^{2}+x+1\right) \mathrm{e}^{-x}
$$

Diese Kurven zeigt Abbildung 2 mit den Konstruktionslinien zum Auffinden des Konzentrationsintervalles mit Linearitätsabweichungen innerhalb der zugelassenen Grenzen. Dieses Intervall erstreckt sich über $56 \%$ von $c_{0}$ und ist mit dem aus Abbildung 1 für ein Drei-Kammer-System entnommenen Wert $46 \%$ zu vergleichen.

Mit vier Mischkammern in Serie erhält man gemäss Gleichung (11):

$$
\Delta c_{1}\left(x^{3} / 6\right)+\left(\Delta c_{2}-\Delta c_{1}\right)\left(x^{2} / 2\right)+\left(\Delta c_{3}-\Delta c_{2}\right) x+\left(\Delta c_{4}-\Delta c_{3}\right)=0
$$

Diese Gleichung ist für gewünschte Inflexionspunkte bei $x=0,1$ und 2 mit $x(x-1)(x-2)=0$ zu identifizieren. Dies führt zu den Beziehungen:

$$
\Delta c_{4}=\Delta c_{3} \quad \Delta c_{2}=0 \quad \Delta c_{1}=3 \Delta c_{4}
$$

und zu den folgenden möglichen Konzentrationsreihen:

$$
5,2,2,1,0 \quad \text { und } 0,3,3,4,5
$$

Die entsprechenden Gleichungen für die Konzentration und deren Gradienten sind (für den positiven Gradienten):

$$
\begin{aligned}
& c_{4}(x)=c_{0}-0,1 \quad c_{0}\left(x^{3}+3 x^{2}+8 x+10\right) \mathrm{e}^{-x} \\
& c_{4}{ }^{\prime}(x)=0,1 \quad c_{0}\left(x^{3}+2 x+2\right) \mathrm{e}^{-x}
\end{aligned}
$$




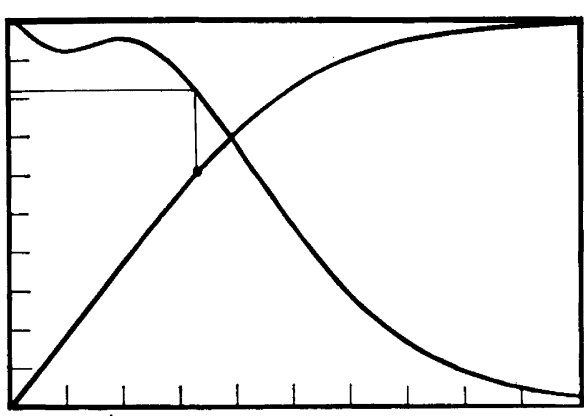

$A b b$. 3. Ausflusskonzentration (steigende Kurve) und deren Gradient von einem Kammersystem mit vier Kammern als Funktion des gelieferten Volumens, für den Fall, dass die fünf Ausgangskonzentrationen den Zahlen 5, 2, 2, 1 und 0 proportional sind. (Vgl. die Gleichungen 17 bis 21.)

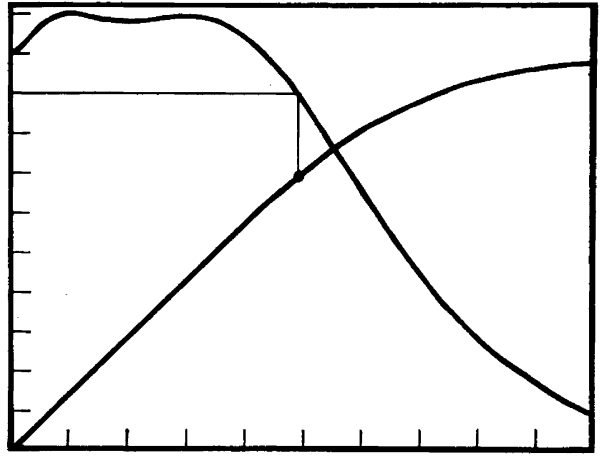

Abb. 4. Ausflusskonzentration (steigende Kurve) und deren Gradient von einem Kammersystem mit fünf Kammern als Funktion des gelieferten Volumens, für den Fall, dass die sechs Ausgangskonzentrationen den Zahlen 15, 3, 9, 4, 2 und 0 propor. tional sind. (Vgl. die Gleichungen 22-23).

Diese Funktionen werden in Abb. 3 graphisch dargestellt. Man sieht, dass man eine genügende Linearität bis zu $0,62 c_{0}$ erhält, im Vergleich zu $0.525 c_{0}$ für Ausgangskonzentrationen in aritmetischer Reihe.

Berechnungen gleicher Art für fünf Kammern und mit Inflexionspunkten bei $x=0,1,2$ und 3 ergeben die Konzentrationsfolgen:

$$
15,3,9,4,2,0 \text { und } 0,12,6,11,13,15
$$

Die erste Folge (steigende Konzentration) entspricht den Funktionen:

$$
\begin{aligned}
& c_{5}(x)=c_{0}-\left(c_{0} / 30\right)\left(x^{4}+2 x^{3}+11 x^{2}+26 x+30\right) \mathrm{e}^{-x} \\
& c_{5}{ }^{\prime}(x)=\left(c_{0} / 30\right)\left(x^{4}-2 x^{3}+5 x^{2}+4 x+4\right) e^{-x}
\end{aligned}
$$

Diese Kurven werden in Abb. 4 gezeigt. Der den Anforderungen genügende Linearitätsumfang ist jetzt auf $0,695 c_{0}$ angewachsen, verglichen mit dem Wert 0,57 $c_{0}$ bei Abwesenheit von Inflexionspunkten (Abb. 1).

Bei einem Sechs-Kammer-System können die Inflexionspunkte auf die Werte $x=0,1,2,3$ und 4 verlegt werden, woraus die Konzentrationsfolgen:

$$
60,0,60,15,20,13,6 \text { und } 0,60,0,45,40,47,54
$$

errechnet werden. In diesem Falle umfasst der Gradient 54 Konzentrationseinheiten, während 60 Einheiten zu dessen Aufbau notwendig sind. Die Konzentration:

$$
c_{6}(x)=c_{0}-\left(c_{0} / 120\right)\left(x^{5}+15 x^{3}+40 x^{2}+94 x+108\right) \mathrm{e}^{-x}
$$

und ihr Gradient

$$
c_{6}{ }^{\prime}(x)=\left(c_{0} / 120\right)\left(x^{5}-5 x^{4}+15 x^{3}-5 x^{2}+14 x+14\right) e^{-x}
$$

sind graphisch in Abb. 5a dargestellt. Wie man aus dieser Abbildung entnehmen kann, ist der Gradient über etwa 4 Kammervolumina bis auf $\pm 1 \%$ Acta Chem. Scand. 16 (1962) No. 9 


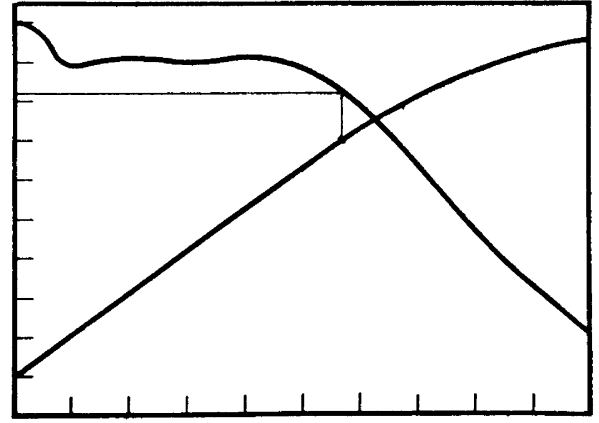

Abb. 5a. Ausflusskonzentration (steigende Kurve) und deren Gradient von einem Kammersystem mit sechs Kammern als Funktion des gelieferten Volumens, für den Fall, dass die sieben Ausgangskonzentrationen den Zahlen 60, 0, 60, 15, 20, 13 und 6 proportional sind. (Vgl. die Gleichungen 25-26.)

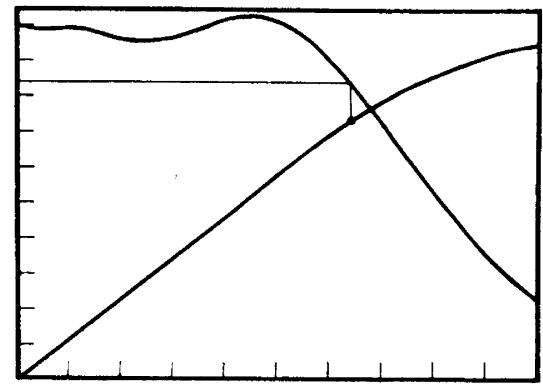

$A b b .5 b$. Ausflusskonzentration (steigende Kurve) und deren Gradient von einem Kammersystem mit sechs Kammern als Funktion des gelieferten Volumens, für den Fall, dass die sieben Ausgangskonzentrationen den Zahlen 984, 24, 696, 300, 230, 115 und 0 proportional sind. (Vgl. die Gleichungen 27 -28.)

konstant. Die Grenze für eine $\pm 10 \%$-ige Abweichung ist aber nur $0,70 c_{0}$, also nicht besser als für das Fünf-Kammer-System. Ohne Inflexionspunkte (Abb. 1) erreicht man 0,60 $c_{0}$.

Versucht man, um den Linearitätsbereich noch zu erweitern, die Inflexionspunkte auf zu hohe $x$-Werte zu legen, so ergeben die Identitäten manchmal negative Konzentrationen. Es ist aber durchaus möglich, den Linearitätsbereich durch Verlegung gewisser Inflexionspunkte zwischen ganzzahlige $x$ Werte zu verbessern. Falls man z. B. in einem Sechs-Kammer-System die Inflexionspunkte an $x=0,1 / 2,1,5 / 2$ und $9 / 2$ verlegt, so bekommt man für eine wachsende Ausflusskonzentration die folgenden Ausgangskonzentrationen: $984,24,696,300,230,115,0$

welche der Ausflusskonzentration:

$$
c_{6}(x)=c_{0}-\left(c_{0} / 984\right)\left(8 x^{5}+12 x^{4}+114 x^{3}+377 x^{2}+869 x+984\right) \mathrm{e}^{-x}
$$

und dem Ausflussgradienten:

$$
c_{6}{ }^{\prime}(x)=\left(c_{0} / 115\right)\left(8 x^{5}-28 x^{4}+66 x^{3}+35 x^{2}+115 x+115\right) \mathrm{e}^{-x}
$$

entsprechen. Diese Kurven werden in Abb. $5 b$ gezeigt. Man sieht dort, dass der Gradient bis zu 0,735 $c_{0}$ eine $\pm 10 \%$-ige Konstanz besitzt.

\section{BESCHREIBUNG DER APPARATUR}

Konstruktive Gesichtspunkte. Wesentlich für die Reproduzierbarkeit und gute Übereinstimmung der Ausflusskonzentrationen mit der Theorie sind hauptsächlich Volumengleicheit der Kammern und eine wirksame Durchmischung des Kammerinhaltes. 
Die erstgennante Forderung ist durch Einhalten enger Toleranzen bei Herstellung und Bearbeitung der Kammerbestandteile weitgehendst erfüllbar. Der Durchmischungsgrad des Kammerinhaltes ist von einer Reihe verschiedener Faktoren abhängig. Abgesehen von Viskositäts- und Hafteigenschaften der Flüssigkeit sind einige dieser Faktoren für die Gestaltung und Dimensionierung der Apparatur von Bedeutung. Insbesondere gilt dies die Form der Rührkörper und deren relative Bewegung zu den Kammerwänden.

Die Aneinanderschaltung kapillar verbundener und mit Rührwerk versehener Kammern kan konstruktiv verschieden durchgeführt werden. Mit Hinsicht auf die erwähnte relative Bewegung zwischen Rührkörpern und Kammerwandung kann man zwei Prinzipien unterscheiden. Einerseits ein stillstehendes Kammersystem mit einer durch sämtliche Kammern geführten Achse, an welcher die Rührkörper beispielweise ausschwenkbar befestigt sind, oder ein um seine eigene Achse rotierendes Kammersystem, wobei die Kammern mit freiliegenden, rollenden Rührkörpern, wie Kugeln, Zylindern, oder dergleichen versehen sind. Im ersteren Falle ist die Ab-und Zuführung der Flüssigkeit leicht durch festen Anschluss an die Endkammern zu bewerkstelligen. Schwieriger gestaltet sich die Konstruktion des Rührwerkes und dessen Lagerung. Bei rotierenden Kammern dagegen ist die Ab- und Zuführung der Flüssigkeit schwieriger.

Ein Aufbau des Kammersystemes aus einfachen Komponenten, wie plattenförmigen Trennungswänden zwichen rohrförmigen Kammerelementen gestattet zum Zwecke der Reinigung ein leichtes Auseinandernehmen und Zusammensetzen. Eine weitere wertfolle Folge einer solchen Konstruktion liegt darin, dass Rührkörper verschiedener Grösse angewendet werden können. Diese erhalten hierdurch eine doppelte Aufgabe, teils die der Durchmischung und teils die der Änderung des freien Kammervolumens. In gewissen Grenzen kann also mit demselben Apparat durch Wahl verschieden grosser Rührkörper der Gradient über ein gewünschtes Flüssigkeitsvolumen verteilt werden.

Ausführung. Wir haben die Konstruktionsalternative der rotierenden Kammern gewählt und die Frage des $\mathrm{Ab}$ - und Zuflusses so gelöst, dass wir als Lagerung des Kammersystemes gewöhnliche Kugelschliffe mit kapillarem Durchfluss anwenden. Die Abbildungen 6-9 zeigen Gesamtansicht der betriebsfertigen Apparatur, Gesamtansicht der Apparatur mit zur Füllung aufgestelltem Kammersystem, Teilansicht des Kammersystemes mit Hinsicht auf den Kammeraufbau, und Teilansicht je einer Zwischen- und Endkammerplatte. Die konstruktiven Einzelheiten sind aus den Figurtexten zu entnehmen.

Die Kugelpfannen $(9, \mathrm{~s})$ sind in die Aussenwände der Endkammern $(6, f, q)$ versenkt, während die Kugeln mit ihren Stutzen (6, a, v), durch Gummihülsen geschützt, in Lagerböcken $(7, b, u)$ festgeschraubt sind. Der eine dieser Lagerböcke $(7, b)$ ist auf der Grundplatte $(7, y)$ in einer Führung $(7, z)$ verschiebbar ausgeführt. Der Lager- und Abflusstutzen $(7, a)$ ist gegen den Lagerbock $(7, b)$ federnd gelagert. Die Verschiebbarkeit des Lagerbockes $(7$, b) gewährt ein leichtes Einsetzen und Herausnehmen des Kammersystems, sowie die Möglichkeit, die Anzahl der Kammern zu variieren. Die Federung $(7, c)$ garantiert einen gleichartigen Lagerdruck. Mit einer dünnen Schicht hochwertigen Fettes in den Schliffen haben wir selbst bei mehrtägigem dauerndem Gang des Apparates keinen Flüssigkeitsaustritt an den Lagerstellen beobachten können. Auch 


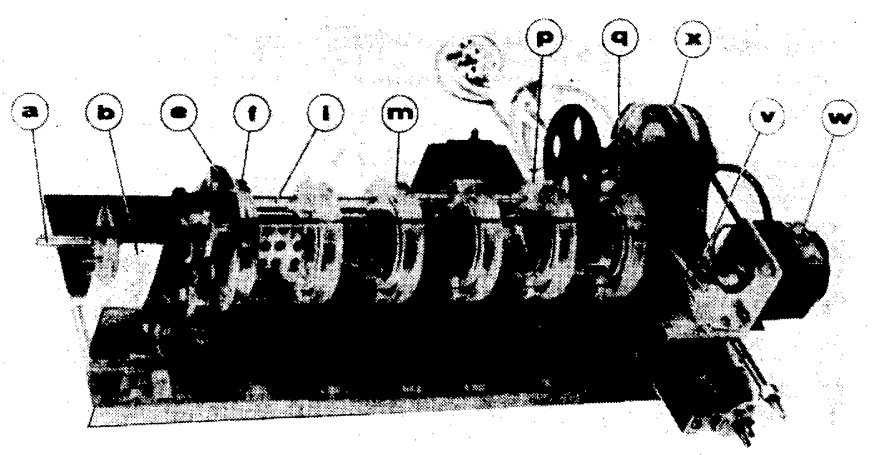

$A b b, 6$.

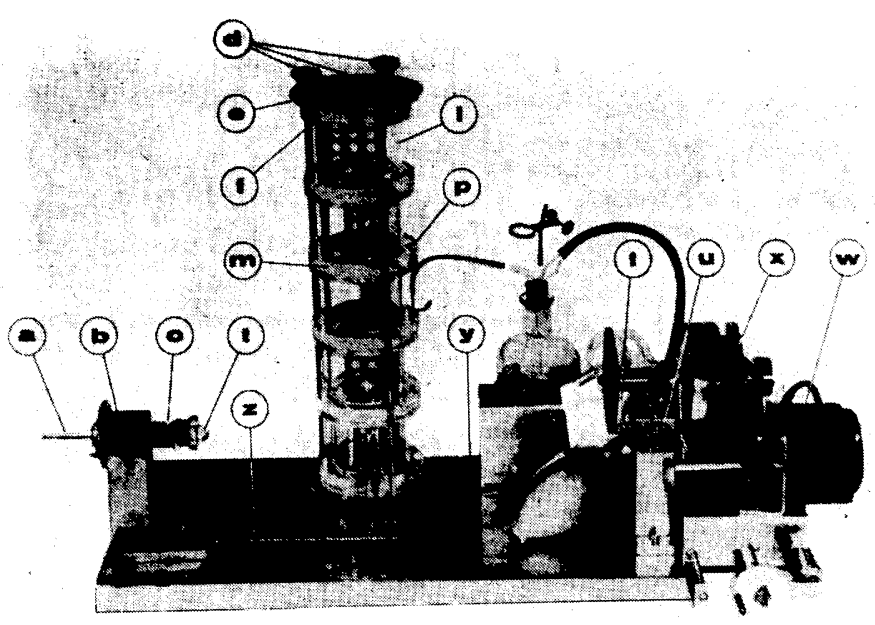

$A b b . \%$.

der befürchtete Verschleiss der Schliffe ist trotz der jetzt schon mehrjährigen Anwendung nicht feststellbar.

Die Kammern bestehen aus losen Rohrstücken (8, i), Platten (9), und Rührkörpern $(8, \mathrm{~h})$. Jede Platte, mit Ausnahme der beiden Endplatten $(6, f, q)$, bildet Scheidewand zwischen zwei Kammern und ist in der Mitte kapillar durchbohrt $(9, \mathrm{n})$. Die beiden Endplatten $(6 \mathrm{f}, \mathrm{q})$ tragen, wie oben erwähnt, die Pfannen der Lagerkugelschliffe. Dichten und Zentrieren der Kammern gegeneinander geschieht selbsttätig durch ringförmige Nuten $(9, \mathrm{k})$ in den Plattenseiten. Diese Nuten sind dimensionsmässig an die Enden der rohrförmigen Kammerelemente angepasst. Das Füllen der Kammern geschieht durch kapillare Bohrungen $(9$, o) durch die Platten von aussen (7). Diese Kanäle 


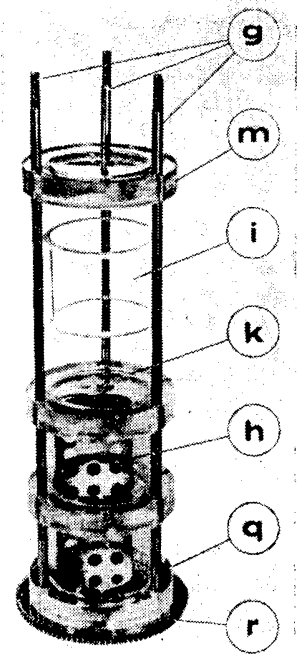

Abb. 8 .

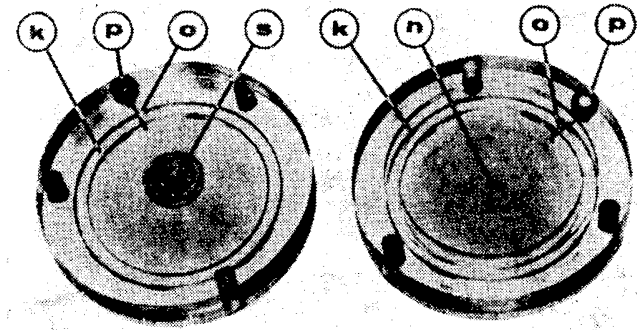

Abb. 9.

$A b b$. 6. Gesamtansicht der betriebsfertigen Apparatur.

$A b b$. 7. Gesamtansicht der Apparatur mit zur Füllung aufgestelltem Kammersystem.

Abb. 8. Teilansicht des Kammersystemes mit Hinsicht auf den Kammeraufbau.

$A b b$. 9. Teilansicht je einer Zwischen- und Endkammerplatte.

Die Buchstaben in der Abbildungen 6-9 bezeichnen: a) Abflusstutzen mit Lagerkugelschliff. b) Verschiebbarer Lagerbock. c) Feder zur Einstellung des Lagerdruckes. d) Gerändelte Spannmuttern. e) Metallspannplatte. f) Endplatte gegen Abflusseite. g) Spannstangen. h) Rührkörper. i) Rohrförmiges Kammerelement. k) Ringförmige Steuernuten. m) Plattenförmige Kammerzwischenwand. n) Kapillare Durchflussbohrung in der Kammermitte. o) Verchliessbare Einfüllöffnung. p) Konischer Verschlusstopfen. q) Endplatte gegen Antriebseite. r) Als Zahnrad ausgeformte Metallspannplatte. s) Pfannenteil der Kugelschlifflagerung. t) Kugelteil der Kugelschlifflagerung. u) Fester Lagerbock. v) Zuflusstutzen mit Lagerkugelschliff. w)

Motor. x) Getriebe. y) Grundplatte. z) Führungsnute für verschiebbaren Lagerbock.

werden nach erfolgter Füllung durch Drehung eines durchbohrten Stopfens $(9, p)$, der wie ein Hahnküken wirkt, verschlossen. Eine solche Verschlusskonstruktion führt keine Druckänderung oder sonstige Einflüsse auf den Kammerinhalt mit sich.

Wir haben erst mit sechs und später mit fünf Kammern gearbeitet, da sich eine solche Beschränkung der Kammeranzahl für uns als vollauf genügend erwies, um in den aufgestellten Grenzen einen konstanten Gradienten zu erreichen. Jede Kammer hat einen grössten freien Rauminhalt von $100 \mathrm{~cm}^{3}$. Der hierbei angewendete Rührkörper besteht aus zwei Ringen, welche mittels einer vielfach durchbohrten rechteckigen Platte zentrisch so verbunden sind, dass sie miteinander parallel und winkelrecht zur Verbindungsplatte stehen.

Acta Chem. Scand. 16 (1962) No. 9 
Diese auf den Ringen rollenden Rührkörper $(8, \mathrm{~h})$ sind aus Messing gefertigt und nachträglich hartvergoldet. Die grössten Rührkörper sind im Wesentlichen zylindrisch. Zum Ausschluss von Luftblasen bei Füllung der Kammern wurden die Plattenseiten konisch ausgearbeitet und die Füllanschlüsse so plaziert, dass das Kammersystem während der Füllung vertikal steht.

Der Dichtungsdruck der Kammern gegeneinander wird durch Spannstangen $(8, g)$ gewährleistet, welche mit Metallendplatten ( 7, e; $8, \mathrm{r})$ zusammenwirken und mit Hilfe von gerändelten Muttern $(7, d)$ angezogen werden. Die eine der Metallendplatten $(8, \mathrm{r})$ ist als Zahnrad zum Antrieb des Systemes mittels Motor $(6, \mathrm{w})$ und Getriebe $(6, \mathrm{x})$ ausgeformt. Aus Gründen der Zeitersparnis und für einen schnellen Nachweis der Übereinstimmung mit der Theorie wurde die hier beschriebene Apparatur aus Plexiglas hergestellt. Es ist jedoch eine Glaskonstruktion auf demselben Prinzip unter Arbeit.

Handhabung. Die Zusammensetzung des Kammersystemes geschieht durch abwechselndes Aneinanderreihen von platten- und rohrförmigen Kammerelementen. Man beginnt den Aufbau mit der Zahnradspannplatte $(8, r)$ und den von dieser getragenen Spannstangen $(8, g)$. Die eine Endplatte (8, q) wird, von den Spannstangen gefürt, so angesetzt, dass die Kugelpfanne gegen das Zahnrad gewendet ist. Hierauf wird eines der rohrförmigen Kammerelemente $(8$, i) mit einem der offenen Enden in die Zentriernute $(9, \mathrm{k})$ der Endplatte $(8, \mathrm{q})$ gepresst. Zum Zwecke der sicheren Abdichtung kann man vor der Zusammensetzung des Kammersystems sämtliche Zentriernuten in den Plattenelementen mit dünnen Dichtungsringen aus einem passenden Material, wie Polyaethylen, Polytetrafluoraethylen o. dgl. versehen. Nach Ansatz des Rohrelementes wird ein Rührkörper eingelegt und hierauf eine der Zwischenplatten $(8, \mathrm{~m})$, von den Spannstangen geführt, so aufgesetzt, dass die untere Zentriernute der Platte das darunterliegende offene Rohrende erfasst, steuert und abdichtet. Hierbei ist darauf zu achten, dass die Einfüllanordnung mit dem konischen Stopfen $(7, \mathrm{p})$ so gerichtet ist, dass in jede auf beschriebene Weise entstehende Kammer eine Einfüllöffnung mündet.

Da die Zwischenplatten $(8, \mathrm{~m})$ auf beiden Seiten mit Zentrier- und Dichtungsnuten $(9, \mathrm{k})$ versehen sind, kann der Aufbau der übrigen Kammern auf analoge Weise fortgesetzt werden, bis schliesslich die letzte Kammer mit der Endplatte (7, f) abgeschlossen wird. Die Kugelschlifflagerpfanne dieser Endplatte ist dabei nach aussen zu wenden, wobei die Zentriernute das letzte offene Rohrende abdichtet. Das ganze Kammersystem wird dann mit der Metallspannplatte $(7$, e) abgeschlossen, und die gerändelten Spannmuttern $(7, d)$ werden aufgesetzt und angezogen. Es ist vorteilhaft, diesen Aufbau vertikal durchzuführen, insbesondere da das System in dieser Stellung gefüllt wird. Vor der Füllung ist die kapillare Öffnung der in der vertikalen Stellung unteren Kugelpfanne mit einem kleinen Stopfen, z. B. einer Zahnstocherspitze, zu verschliessen, um das Austreten der Flüssigkeit während der Füllung zu verhindern. Die vorbereiteten Lösungen der gewählten Konzentrationsreihe werden, von der untersten Kammer angefangen, in ihre Kammern eingefüllt, wobei man sich entweder des Überdruckes der Flüssigkeit in einem Einfüllröhrchen mit Trichter bedient oder, wie aus Abb. 7 hervorgeht, eines erzeugten Luftüberdruckes. Sobald die Flüssigkeit beginnt, die obere Abschlussfläche einer Kammer zu benetzen, wird die Zuflussgeschwindigkeit soweit herabgesetzt, dass man imstande ist, den Zufluss augenblicklich zu unterbrechen. Dies geschieht, wenn die Flüssigkeit die kapillare Bohrung zur darüberliegenden leeren Kammer gerade füllt, ohne in diese Kammer einzutreten. Der Ausschluss von Luftblasen und die Beobachtung des Flüssigkeitsspiegels bei Eintritt in die obere kapillare Bohrung einer Kammer wird wesentlich von der konischen Ausführung der Plattenseiten begünstigt. Nach orfolgter Füllung des ganzen Kammersystemes wird der Lager- und Zuflusstutzen (6, v) mit der Vorratsflüssigkeit gefüllt und luftfrei mit dem Vorratsgefäss oder dem Pumpensystem der Vorratslösung verbunden. Dies ist notwendig, um das Eindringen von Luft in das Kammersystem zu verhindern. Der verschiebbare Lagerbock $(7, \mathrm{~b})$ wird in die äusserste Lage geführt, worauf, nach Abdichtung der oberen Kugelpfanne, der Verschlusstopfen der unteren Kugelpfanne entfernt werden kann. Jetzt kann das Kammersystem an den festen Lagerbock $(7, \mathrm{u})$ angesetzt und in die Horizontallage überführt werden. Nach 
Abb. 10. Vergleich der Ausflusskonzentrationen mit den theoretisch berechneten Werten. Gemessene Konzentrationen: $O$. Berechnete Konzentrationen: - +. Die obere Kurve wurde mit einem Sechs-KammerSystem erhalten, in welchem die Ausgangskonzentrationen 0 (Vorratslösung), 1, 2, 3, 4,5 und 6 Millimol Natriumbenzoat per Liter waren. Die untere Kurve rührt von einem Fünf-Kammer-System her mit den Ausgangskonzentrationen $0,1,2,3,4$ und 5 Millimol per Liter.

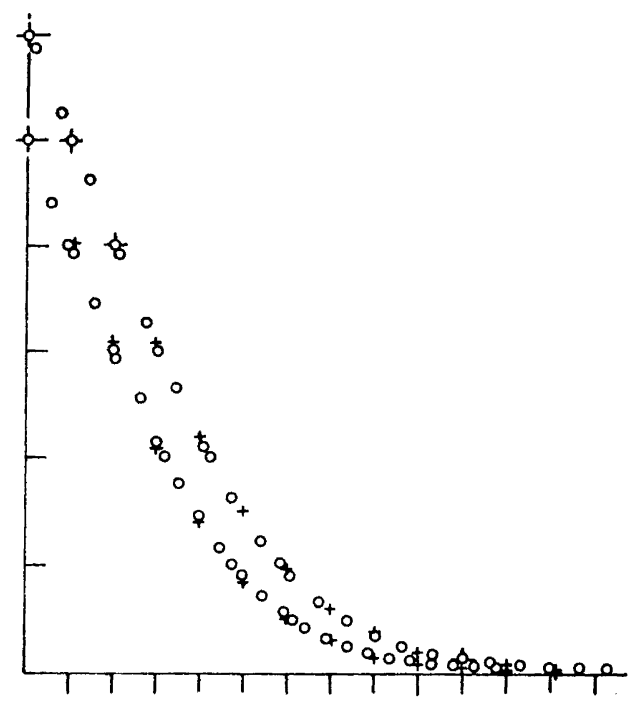

Entfernung der Abdichtung der Kugelpfanne kann der verschiebbare Lagerbock (7, b) mit dem federnd gelagerten Abflusschliff $(7, a)$ zugeschoben und verriegelt werden. Der Lagerdruck wird von der Feder $(7$, c) bestimmt und ist durch kleine Verschiebungen des Lagerbockes (7, b) leicht zu regulieren. Der Abflusstutzen (7, a) wird an die Kolonne, in welcher der Gradient angewendet werden soll, angeschlossen, und der Motor $(6, \mathrm{w})$ in Betrieb gesetzt. Je nach Art der Flüssigkeit und Rührkörper kann mit Hilfe des Getriebes $(6, \mathrm{x})$ eine optimale Durchmischung erreicht werden.

Erst wenn das Kammersystem rotiert, wird die Zuleitung der Vorratslösung geöffnet. Der notwendige Druck kann von einem höherliegenden Vorratsgefäss oder von einer Pumpe vermittelt werden.

Nach erfolgter Überführung der gewünschten Flüssigkeitsmenge zur Kolonne wird zuerst der Zufluss zum Kammersystem gesperrt und der Motor (6, w) abgeschaltet. Darauf erst wird die Zuleitung zur Kolonne gesperrt. Jetzt kann das Kammersystem auseinandergenommen werden. Dies geschieht am besten in umgekehrter Reihenfolge zum Aufbau. Unter Verschiebung des Lagerbockes (7, b) wird das Kammersystem gegen den festen Lagerbock $(7, u)$ gepresst und die freie Kugelpfanne abgedichtet. Hierauf kann auch die andere Kugelpfanne abgedichtet und das System in aufrechte Stellung gebracht werden. Die Spannmuttern (7, d), die Metallspannplatte (7, e), der Verschluss der oberen Kugelpfanne und die Kammerendplatte $(7, \mathrm{f})$ werden in genannter Reihenfolge entfernt. Die obere Kammer liegt jetzt offen und kann mit einer Pipette oder Absaugvorrichtung entleert werden. Hierauf wird das rohrförmige Element und der Rührkörper der obersten Kammer abgenommen und der Abbau der übrigen Kammern analog durchgeführt.

Bei dem beschriebenen Auseinandernehmen des Kammersystemes in aufrechter Stellung hat sich das Eigengewicht der Kammern als genügend erwiesen, um den notwendigen Dichtungsdruck nach Entfernung der Spannmuttern zu liefern, sodass kein unkontrollierter Flüssigkeitsaustritt stattfand. Um ganz sicher zu sein kann man jedoch während des Abbaus den jeweils unter der Abbauebene liegenden Teil der Kammersäule mit einer Hand gegen die Unterlage gedrückt halten, da das Auseinandernehmen schnell und leicht mit der anderen Hand durchgeführt werden kann. 


\section{EXPERIMENTELLES}

Oben beschriebene Mischvorrichtung ist die zweite Ausführungsform gemäss den dargelegten Prinzipien. Die erste Konstruktion wurde im Frühjahr 1960 fertiggestellt, während diese Zweite seit dem Sommer 1961 zur Verfügung stand und häufig in unserem Laboratorium angewendet wurde. Ausserdem haben wir das Instrument mit Hinsicht auf Reproduzierbarkeit und Übereinstimmung der Ausflusskonzentration mit der Theorie kritisch geprüft. Dabei haben wir eine ausserordentlich gute Reproduzierbarkeit gefunden; die Abweichungen liegen unter dem mit gewöhnlichen Methoden Messbaren. Die Experimente zur Prüfung der Ưbereinstimmung mit der Theorie werden hier näher beschrieben.

In einigen Versuchen wurden die Kammern mit Wasserlösungen von $\mathrm{Na}$ triumbenzoat mit Konzentrationen in aritmetischer Reihe gefüllt. Der Ausfluss wurde durch die Küvette des registrierenden UV-Absorptiometers "Uvicord" * geleitet. Für die genannten Lösungen wies die Eichung des Instrumentes strenge Gültigkeit des Lambert-Beer'schen Extinktionsgesetzes in weiten Konzentrationsgrenzen aus. Nichtsdestoweniger wurden die Kurven so aufgenommen, dass vor Beginn und nach Abschluss des Versuches je eine von Anfang separierte Menge Flüssigkeit jeder Ausgangskonzentration registriert wurde. Auf diese Weise konnte eine sich über die ganze Ausflussperiode erstreckende Eichkurve aufgenommen und damit eventuelle systematische Streuungen der registrierenden Apparatur während dieser Periode kompensiert werden. Das Resultat zweier Versuche wird in Abb. 10 gezeigt. Man sieht dort, dass die Übereinstimmung mit der Theorie sehr gut ist. Andere Versuche, die auch Ausgangskonzentrationen zur Erzeugung von Ausflussfunktionen mit Inflexionspunkten umfassten, haben dieses Ergebnis durchaus bestätigt.

Dank. Die Theorie dieser Mischvorrichtung wurde vor einigen Jahren ausgearbeitet. Die Herstellung des Instrumentes und dessen Ausprüfung wurde durch die Einrichtung eines neuen Laboratoriums ermöglicht. Die Verfasser danken dafür der WallenbergStiftung, der Wenner-Green Gesellschaft und Magnus Bergvalls Stiftung. Die experimentelle Arbeit wurde weiter von den medizinischen, naturwissenschaftlischen und technischen Forschungsräten unterstützt. Für das sorgfältige Berechnen und Zeichnen der Kurven in den Abb. 1-5 danken wir Frl. Kerstin Svensson.

\section{SCHRIFTTUM}

1. Peterson, E. A. und Sober, H. A. Anal. Chem. 31 (1959) 857; "A Laboratory Manual of Analytical Methods of Protein Chemistry, Including Polypeptides", Vol. 1, Eds. P. Alexander and R. J. Block, Pergamon Press, London 1960, p. 88.

2. Svensson, H. "A Laboratory Manual of Analytical Methods in Protein Chemistry, Including Polypeptides" Vol. 1, Eds. P. Alexander and R. J. Block, Pergamon Press, London 1960 , p. 195.

3. Drake, B. Arkiv Kemi 8 (1955) 1.

4. Svensson, H. Science Tools 5 (1958) 37.

Eingegangen am 18. April 1962.

* Hersteller: LKB-Produkter, Box 12220, Stockholm 12. 